lipid-lowering treatments. Coronary artery bypass grafting (CABG) is commonly used to bypass coronary arteries diseased by atherosclerosis, routinely using the saphenous vein (SV) as a conduit. Early after grafting the SV adapts to the arterial environment through reendothelialisation, and increased motility of smooth muscle cells (SMC). A clinical association between $\mathrm{Lp}(\mathrm{a})$ and coronary artery disease is evident; however, its role in vein graft failure is less clear. Endothelial cells (EC) and SMC were cultured from the SV of patients undergoing CABG. The influence of apo(a) on cellular activity was examined by proliferation (cell counting), chemotaxis (modified Boyden chamber) and chemokinesis (scratch wound) assays. Apo(a) significantly inhibited SV-EC proliferation $(n=9$, $\mathrm{p}<0.001$ ). Although no effect on SV-SMC proliferation was apparent, apo(a) markedly modulated SMC motility and appeared to act as a chemorepellent. When SMC were acutely exposed to a gradient of apo(a), they consistently migrated away from the source $(n=6$, $\mathrm{p}<0.01)$. Chronic exposure to apo(a) in the scratch wound model also revealed that the speed of migration was reduced $(n=5, p<0.01)$. Remodelling of the SV is essential for adaptation to an arterial environment, and key to its function as a successful bypass graft. Our studies show that apo(a) inhibits EC proliferation, potentially compromising endothelial repair in the grafted vein. Furthermore, the chemorepellent effect of apo(a) may also impede critical SMC migration required for effective integration. $\operatorname{Lp}(a)$ is therefore likely to contribute to impaired SV adaptation and inferior graft patency.

\section{BAS/ CRUCIAL ROLES OF CBX3 IDENTIFIED BY NUCLEAR BSCR30 PROTEOMICS IN SMOOTH MUSCLE DIFFERENTIATION FROM STEM CELLS AND VASCULAR INJURY-INDUCED NEOINTIMA FORMATION}

\section{doi:10.1136/hrt.2010.205781.41}

\begin{abstract}
${ }^{1,2} \mathrm{O}$ Xiao, ${ }^{1} \mathrm{G}$ Wang, ${ }^{1} \mathrm{X}$ Bai, ${ }^{1} \mathrm{X}$ Yin, ${ }^{1} \mathrm{Z}$ Luo, ${ }^{1} \mathrm{M}$ Mayr, ${ }^{2} \mathrm{~S}$ Ye, ${ }^{1} \mathrm{O} \mathrm{Xu} .{ }^{1}$ Cardiovascular Division, King's College London BHF Centre, London, UK; ${ }^{2}$ Clinical Pharmacology, William Harvey Research Institute, Barts and The London School of Medicine and Dentistry, Queen Mary University of London, London, UK
\end{abstract}

Rationale Our previous studies have developed an efficiency method for producing a large number of smooth muscle cells (SMCs) from embryonic stem (ES) cells. However, little is known about the underlying mechanism.

Methodology and results Nuclear proteins were harvested and isolated from undifferentiated and differentiating ES cells at different time points, and subjected to proteomics analysis. Notably, the majority of upregulated nuclear proteins during SMC differentiation were involved in chromatin remodelling, cellular morphogenesis, cell proliferation, DNA replication, protein synthesis, mRNA transport and RNA processing processes. We further focused on chromobox protein homologue 3 ( $\mathrm{Cbx} 3$ ) owing to its involvement in the regulation of gene-specific expression. Knockdown of $\mathrm{Cbx} 3$ in the differentiating ES cells resulted in downregulation of smooth muscle differentiation markers, while enforced expression of this gene enhanced SMC differentiation in a dose-dependent manner. Our data also suggested that $\mathrm{Cbx} 3$ mediates SMC differentiation from ES cells through regulation of smooth muscle-specific transcription factor, serum response factor (SRF) and its coactivator myocardin. Furthermore, we also demonstrated that another smooth muscle transcription factor, Dia1, functions as bridge protein between $\mathrm{Cbx3}$ and SRF, through which Cbx3 modulates SRF activation, and mediates ultimately SMC differentiation from stem cells. Importantly, in vivo perivascular knockdown of Cbx3 significantly increased wire-injuryinduced neointima formation in mice.

Conclusions Our findings demonstrated for the first time that $\mathrm{Cbx} 3$ has a crucial role in SMC differentiation and possesses an important protective function in vessel injury-induced neointima formation, indicating that $\mathrm{Cbx} 3$ could be a potential new therapeutic target for intervention in SMC proliferative-related vascular diseases.

\section{BAS/ METABOLIC HOMOEOSTASIS IS MAINTAINED IN BSCR31 MYOCARDIAL HIBERNATION BY ADAPTIVE CHANGES IN THE TRANSCRIPTOME AND PROTEOME}

doi:10.1136/hrt.2010.205781.42

${ }^{1}$ Anna Zampetaki, ${ }^{2} \mathrm{D}$ May, ${ }^{2} \mathrm{G}$ Oren, ${ }^{1} \mathrm{X}$ Yin, ${ }^{1} \mathrm{O} \mathrm{Xu},{ }^{3} \mathrm{~A}$ Horrevoets, ${ }^{2} \mathrm{E}$ Keshet ${ }^{1} \mathrm{M}$ Mayr. ${ }^{1}$ King's BHF Centre, King's College London, UK; ${ }^{2}$ Hebrew University, Jerusalem, Israel; ${ }^{3} \mathrm{VU}$ University Medical Center, Amsterdam, The Netherlands

Rationale We have recently established a transgenic mouse model for conditional induction of long-term hibernation via myocardiumspecific induction of a VEGF-sequestering soluble receptor.

Objective Using a combined '-omics' approach, we aim to resolve the cardioprotective response that preserves myocardial viability under chronic hypoxia by integrating mRNA, protein and metabolite changes in unsupervised network analysis.

Methods and results A genome array, difference in gel electrophoresis and proton nuclear magnetic resonance spectroscopy were employed to dissect the hibernation process into an initiation and a maintenance phase. The initiation phase was characterised by peak levels of K(ATP) channel and glucose transporter 1 (GLUT1) expression. Glibenclamide, an inhibitor of K(ATP) channels, blocked GLUT1 induction. In the maintenance phase, tissue hypoxia and GLUT1 expression were reduced and metabolite concentrations were kept relatively constant. Unguided bioinformatics analysis on the combined datasets confirmed that anaerobic glycolysis was affected and that the observed enzymatic changes in cardiac metabolism were directly linked to hypoxia-inducible factor (HIF)-1 activation. Notably, the combination of the proteomic and transcriptomic datasets improved the statistical confidence of the pathway analysis by two orders of magnitude, with HIF-hypoxia-Akt signalling and glycolysis being the most significant.

Conclusions We demonstrate how combining different '-omics' datasets aids in the identification of key biological pathways: chronic hypoxia resulted in a pronounced adaptive response at the transcript and the protein level to keep metabolite levels steady. This preservation of metabolic homoeostasis is likely to contribute to the long-term survival of the hibernating myocardium.

\section{BAS/ NICOTINIC ACID ADENINE NUCLEOTIDE PHOSPHATE IS BSCR32 INVOLVED IN ISCHAEMIA-REPERFUSION-INDUCED CA2+ OSCILLATIONS AND CELL DEATH}

doi:10.1136/hrt.2010.205781.43

N S Tan, S M Davidson, D M Yellon. The Hatter Cardiovascular Institute, University College London Hospital \& Medical School, UK

Reperfusion of ischaemic cells causes intracellular $\mathrm{Ca}^{2+}$ oscillations as the sarcoplasmic reticulum (SR) takes up and releases $\mathrm{Ca}^{2+}$, leading to hypercontracture and cell death. In other systems, nicotinic acid adenine nucleotide phosphate (NAADP) acts as a second messenger to stimulate $\mathrm{Ca}^{2+}$ release from acidic intracellular $\mathrm{Ca}^{2+}$ stores, which in turn triggers $\mathrm{Ca}^{2+}$ release from the SR. We hypothesised that NAADP signalling is involved in the $\mathrm{Ca}^{2+}$ fluctuations that occur at reperfusion.

We examined the effects of a novel NAADP inhibitor, Ned-19, on ischaemia-reperfusion injury in isolated adult rat ventricular cardiomyocytes (ARVC). The sensitivity of mitochondrial permeability transition pore (mPTP) was measured in ARVC using a laser-induced 